Kansas State University Libraries

New Prairie Press

\title{
USING FUNCTIONAL DATA ANALYSIS TO EVALUATE EFFECT OF SHADE ON BODY TEMPERATURE OF FEEDLOT HEIFERS DURING ENVIRONMENTAL HEAT STRESS
}

F. Yang

A. M. Parkhurst

C. N. Lee

T. M. Brown-Brandl

P. E. Hillman

See next page for additional authors

Follow this and additional works at: https://newprairiepress.org/agstatconference

Part of the Agriculture Commons, and the Applied Statistics Commons (c) (1) (3)

This work is licensed under a Creative Commons Attribution-Noncommercial-No Derivative Works 4.0 License.

\section{Recommended Citation}

Yang, F.; Parkhurst, A. M.; Lee, C. N.; Brown-Brandl, T. M.; and Hillman, P. E. (2013). "USING FUNCTIONAL DATA ANALYSIS TO EVALUATE EFFECT OF SHADE ON BODY TEMPERATURE OF FEEDLOT HEIFERS DURING ENVIRONMENTAL HEAT STRESS," Conference on Applied Statistics in Agriculture.

https://doi.org/10.4148/2475-7772.1022

This is brought to you for free and open access by the Conferences at New Prairie Press. It has been accepted for inclusion in Conference on Applied Statistics in Agriculture by an authorized administrator of New Prairie Press. For more information, please contact cads@k-state.edu. 


\section{Author Information}

F. Yang, A. M. Parkhurst, C. N. Lee, T. M. Brown-Brandl, and P. E. Hillman 


\title{
Using Functional Data Analysis to Evaluate Effect of Shade on Body Temperature of Feedlot Heifers during Environmental Heat Stress
}

\author{
F. Yang ${ }^{1}$, A. M. Parkhurst ${ }^{1}$, C. N. Lee ${ }^{2}$, T.M.Brown-Brandl ${ }^{3}$, P.E. Hillman ${ }^{4}$ \\ ${ }^{1}$ Department of Statistics, University of Nebraska - Lincoln \\ ${ }^{2}$ Department of Human Nutrition, Food and Animal Sciences, University of Hawaii-Manoa \\ ${ }^{3}$ USDA-ARS U.S. Meat Animal Research Center \\ ${ }^{4}$ Department of Biological and Environmental engineering, Cornell University
}

\begin{abstract}
Heat stress can be a serious problem for cattle. Body temperature ( $\mathrm{Tb}$ ) is a good measure of an animal's thermo-regulatory response to an environmental thermal challenge. Previous studies found that $\mathrm{Tb}$ increases in response to increasing ambient temperature in a controlled chamber. However, when animals are in an uncontrolled environment, $\mathrm{Tb}$ is subject to many uncontrolled environmental factors, such as sunshade, wind, and humidity, that increase variation in the data. Hence, functional data analysis (FDA) was applied to analyze the data with uncontrolled environmental factors as curves in the whole series of days in this study. Breed (Angus, MARCIII, MARC-I, Charolais) and availability of shade (access versus no access to sunshade) were included as treatment factors in the statistical model. This study illustrates the potential of FDA to retain all information in the curves. The specific objectives are to use FDA to smooth Tb with large noise, to detect treatment effects on $\mathrm{Tb}$, and to assess the interactions between breed and availability of shade with functional regression coefficients. The results show that FDA can be used to detect significant treatment interactions that may otherwise remain undetected using regular linear or nonlinear models. Significant interactions were found, indicating that access to sun-shade influences the way animals respond to a thermal challenge. Overall, it was found that breeds of cattle with dark-hides were more affected by temperature changes and peak temperatures than breeds of cattle with light-hides. Angus cattle (black) had the highest body temperatures in both shade and no shade areas, while Charolais (white) had the lowest body temperatures in the no shade area. However, MARC III (dark red) experienced the largest temperature differential between shade and no shade. Therefore, breed and availability of shade interactions are important considerations when making predictions to aid in management decisions involving feedlot cattle.
\end{abstract}

Key words: Feedlot Cattle, Fourier Basis, Roughness Penalty, Generalized Cross-validation, Eigenvalues, Permutation Tests, Body Temperature Dynamics 


\section{Introduction}

Beef cattle production is fundamental to the agricultural economy of central US, e.g. Nebraska, Kansas, and Missouri. It is affected by animals' condition and environment. When working in a hot environment, animals suffer heat stress which will reduce growth and may even result in death. Heat stress can be a major cause of lost profits in hostile "production-limiting" regions. Studying heat stress provides a basis for initiation of management practices to improve an animal's well being. Body temperature (Tb) is a good way to measure an animal's thermoregulatory response to the environment. Heat stress in working cattle can be measured as the temperature differential.

Previous studies have discussed the heat stress in a controlled environment. In this study the focus is on heat stress in an uncontrolled environment. We examined body temperatures of animals in a field experiment. Two treatment factors, breed and accessibility to the sunshade, were considered. Functional Data Analysis (FDA) was used to analyze the uncontrolled environmental data. Therefore, the objectives of this study is to apply FDA to smooth data with large noise, to check the breeds and sunshade interaction effects on the $\mathrm{Tb}$, and to align the features of $\mathrm{Tb}$ on heat stress days.

\section{Materials and Methods}

\subsection{Materials and Experimental Design}

A field experimental was conducted over 10 days (from July 27 to Aug 5) in 2004 at USDAARS (Gebremedhin et al., 2008) Meat Animal Research Center Nebraska. Thirty-two heifers from 4 breeds, Angus (A), MARC-III (M3), MARC-I (M1), and Charolais (C), were randomly selected and assign to 4 pens so that 8 heifers ( 2 from each breed) were in each pen. The Angus heifers were all solid black. The MARC III crossbred heifers were mostly dark red (some were black; some were solid, while others had white tailheads and/or white faces). The MARC I heifers were tan. The Charolais heifers were solid white. Shading facilities were set up in two pens so that heifers in these pens had access to shade. Heifers in the remaining pens have no access to shade. (Figure 1) Air temperature (Ta) and body temperature (Tb) were recorded hourly. Due to instrument problems, the records of one Charolais in pen 3 were missing. Therefore, 31 animals were analyzed in this study. Figure 2 (top) shows that Ta followed a sinusoidal pattern over all experimental days and heat stress occurred on days 6,7 , and 8 (i.e. August 1, 2, 3). Large noise was observed in $\mathrm{Tb}$ for all heifers. 
Figure 1. Experimental Design for Four Breeds of Heifers: Angus (A), MARC-III (M3), MARC-I (M1), and Charolais (C),

\begin{tabular}{|c|c|c|c|c|}
\hline & 1 & $\begin{array}{l}\text { Pen } \\
2\end{array}$ & 3 & 4 \\
\hline Shade Accessibility & Shade & Shade & NoShade & NoShade \\
\hline Breeds of Heifers & $\begin{array}{l}\text { A M3 } \\
\text { M1 C } \\
\text { A M3 } \\
\text { M1 C }\end{array}$ & $\begin{array}{l}\text { A } \quad \text { M3 } \\
\text { M1 C } \\
\text { A M3 } \\
\text { M1 C }\end{array}$ & $\begin{array}{l}\text { A M3 } \\
\text { M1 C } \\
\text { A M3 } \\
\text { M1 }\end{array}$ & $\begin{array}{l}\text { A M3 } \\
\text { M1 C } \\
\text { A M3 } \\
\text { M1 C }\end{array}$ \\
\hline
\end{tabular}

Figure 2. Plots of air temperature (Ta) and body temperatures (Tb) for 31 heifers in 4 pens. Heat stress days were on Aug 1-3.

\subsection{Methods}

Commonly, average daily body temperatures are used as a response to "heat". However, it is difficult to find significant effects due to the drawbacks of using the average daily Tb. (Table 3). First, average daily $\mathrm{Tb}$ includes both temperatures at daylight and at night. Second, after averaging, the sinusoidal property of $\mathrm{Tb}$ is ignored. In addition, other factors (i.e. humidity, wind speed, etc.)are also ignored. Thus, average daily $\mathrm{Tb}$ may not adequately represent how breeds and shading would effect $\mathrm{Tb}$. Therefore, although average daily $\mathrm{Tb}$ was easily obtained and compared, it averages out important information and better more accurate ways are available to study $\mathrm{Tb}$ in an uncontrolled environmental. 
Table 3. No significant effects can be found if averaging Tb over days.

\begin{tabular}{|l|l|}
\hline Effects & P-value \\
\hline Shade & 0.9877 \\
\hline Breed & 0.8859 \\
\hline Shade*Breed & 0.3009 \\
\hline
\end{tabular}

Functional data analysis (FDA) provides a good way to analyze quantitative, continuous, periodical, and noisy time series data. (Ramsay et al., 2009) It assumes that the data involves repeated measures of the same process. In FDA, data are transformed to functions and the smoothed functions are used to characterize important features in the data. To transform data, two stages need to be considered: basis functions $\phi_{j}$ and the linear combinations, $\mathrm{y}_{\mathrm{i}}$, of $\phi_{j}$.

$$
\begin{aligned}
& y_{i}=\sum_{j=1}^{2 n+1} c_{j} \phi_{j}\left(t_{i}\right)+\cdots \varepsilon_{i} \\
& \phi_{1}(t)=1 \\
& \phi_{2}(t)=\sin (\omega t) \quad \phi_{3}(t)=\cos (\omega t) \\
& \phi_{4}(t)=\sin (2 \omega t) \quad \phi_{5}(t)=\cos (2 \omega t) \\
& \phi_{n}(t)=\cos (n \omega t) \quad \phi_{n}(t)=\cos (n \omega t)
\end{aligned}
$$

where $\omega=2 \pi / T$ and $\mathrm{T}$ is the period. To determine the linear coefficients $c_{j}$, data were smoothed by a roughness penalty.

The choice of roughness penalty in FDA aims to miss nothing of importance in the data by using a powerful basis expansion, but avoids overfitting the data by imposing a penalty on the "roughness" of the function, where the meaning of "rough" can be adapted to special features of the application from which the data were obtained. The roughness is defined as

$$
\operatorname{PEN}_{m+2}[x]=\int\left[D^{m+2} x(t)\right]^{2} d t
$$

where $D^{m+2} x(t)$ represents the $(\mathrm{m}+2)^{\text {th }}$ derivative of $x(t)$.

The roughness penalty approach uses a large number of Fourier basis functions, but at the same time imposing smoothness by penalizing some measure of function complexity. Raw data were smoothed by minimizing the penalized squared error, which includes SSE and the scaled roughness. It defines a measure of roughness of the fitted curve, and then minimizes a fitting criterion that trades off curve roughness against lack of data fit.

$$
\operatorname{PENSSE}_{\lambda}(x)=[y-x(t)]^{\prime}[y-x(t)]+\lambda \operatorname{PEN}_{2}[x]
$$

The $\lambda$ is a smoothing parameter measuring compromise between fit and smoothness. As $\lambda$ increases, roughness is increasingly penalized and $\mathrm{x}(\mathrm{t})$ will become linear. As $\lambda$ decreases, the penalty is reduced and allows $\mathrm{x}(\mathrm{t})$ to fit the data better. The parameter $\lambda$ can be found by minimizing the generalized cross-validation (GCV). (Craven and Wahba, 1979) The process is 
designed to locate a best value for the smoothing parameter $\lambda$ by minimizing the mean GCV over all animals.

$$
\lambda=\overline{G C V}=\left\{\lambda: \min \left(\left(\frac{n}{n-d f(\lambda)}\right)\left(\frac{S S E}{n-d f(\lambda)}\right)\right)_{i}, i=1 \ldots 31\right\}
$$

After smoothing, we would like to analyze the smoothed functional data beginning with the basic features. The curve registration or feature alignment plays an important role in characterizing features of $\mathrm{Tb}$. Hence, the acceleration of $\mathrm{Tb}$ over time was examined by both frequency (phase) variation and amplitude variation.

After registration, we would like to examine whether the two treatment factors, breed and shade, would have an effect on $\mathrm{Tb}$ during heat stress. To evaluate the treatment effects, functional $\mathrm{F}$ tests and $t$ testscan be conducted. Functional $\mathrm{F} / \mathrm{t}$ tests employ a permutation test methodology. In a functional $\mathrm{T}$ test, the absolute valueof a $t$-statistic at each point was calculated as

$$
T(t)=\frac{\left|\bar{x}_{1}(t)-\bar{x}_{2}(t)\right|}{\sqrt{\frac{1}{n_{1}} \operatorname{var}\left[x_{1}(t)\right]+\frac{1}{n_{2}} \operatorname{var}\left[x_{2}(t)\right]}}
$$

To find a critical value of this statistic, we perform the following procedure:

1. Randomly shuffle the labels of the curves.

2. Recalculate the maximum of $T(t)$ with the new labels.

Repeating this many times allowing a null distribution to be constructed. This provides a reference for evaluating the maximum value of the observed $T(t)$.

In the more general case, i.e. more than two groups of functional data, the same approach can be applied. In this case, we define a functional version of the univariate $F$-statistic:

$$
F(t)=\frac{\operatorname{Var}[\hat{y}(t)]}{\frac{1}{n} \sum_{i=1}^{n}\left(y_{i}(t)-\hat{y}(t)\right)^{2}}
$$

As before, we reduce this to a single number by calculating $\max (F(t))$ and conducting a permutation test. In this case, we permute the response curves (or values), leaving the design unchanged.

\section{RESULTS and DISCUSSION}

\subsection{Smooth the data by Roughness Penalty}

In this study, a Fourier basis with 65 basis functions was used. The GCV for each animal was plotted over $\log _{10}(\lambda)$ from -10 to 20 (Figure 3 ). The minGCV occurs at $\log _{10}(\lambda)=-3$. Hence, the smoothing parameter $\lambda=10^{-3}=0.001$. Tb for all animals were smoothed and shown in Figure 4 . The $\mathrm{Tb}$ of all animals increased after day 6. Most animals suffered high peak $\mathrm{Tb}$ on heat stressed days Aug 1-3. 
Figure 3.Generalized cross-validation(GCV) over $\lambda$ for all animals. The blue bold line -- the mean GCV. Vertical solid line -- $\min ($ Mean GCV)

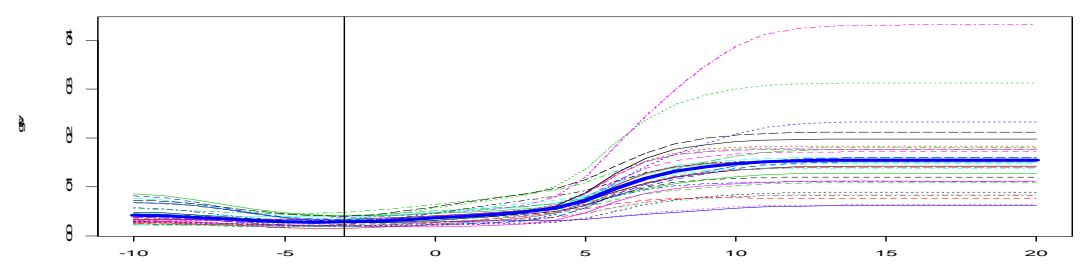

Figure 4.Smoothed hourly $\mathrm{Tb}$ over all days for all 31 animals.

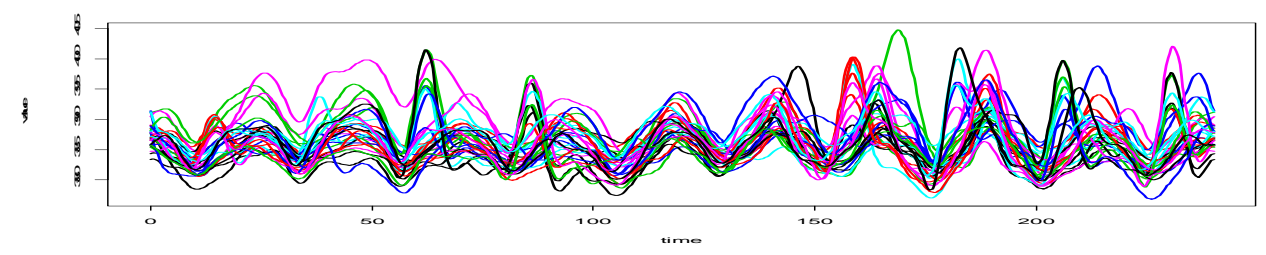

\subsection{Treatment effect}

Functional $\mathrm{F}$ test shows a significant breed*shade interaction effect over all days (Figure 5). Angus in no shade have highest peak Tb over all days, followed by MARC III no shade, Angus shade, MARCI no shade, MARCIII shade, MARCI shade, Charolais no shade, and Charolais shade. Angus (black) in no shade have the largest amplitude of $\mathrm{Tb}$ all the breeds, and Charolais (white) in shade have the smallest amplitude. No matter which breed, heifers always have higher peak $\mathrm{Tb}$ in no shade than in shade. 
Figure 5. Functional $\mathrm{F}$ test for breed * shade interaction effect (top); predicted $\mathrm{Tb}$ in 8 breed * shade combinations (bottom).
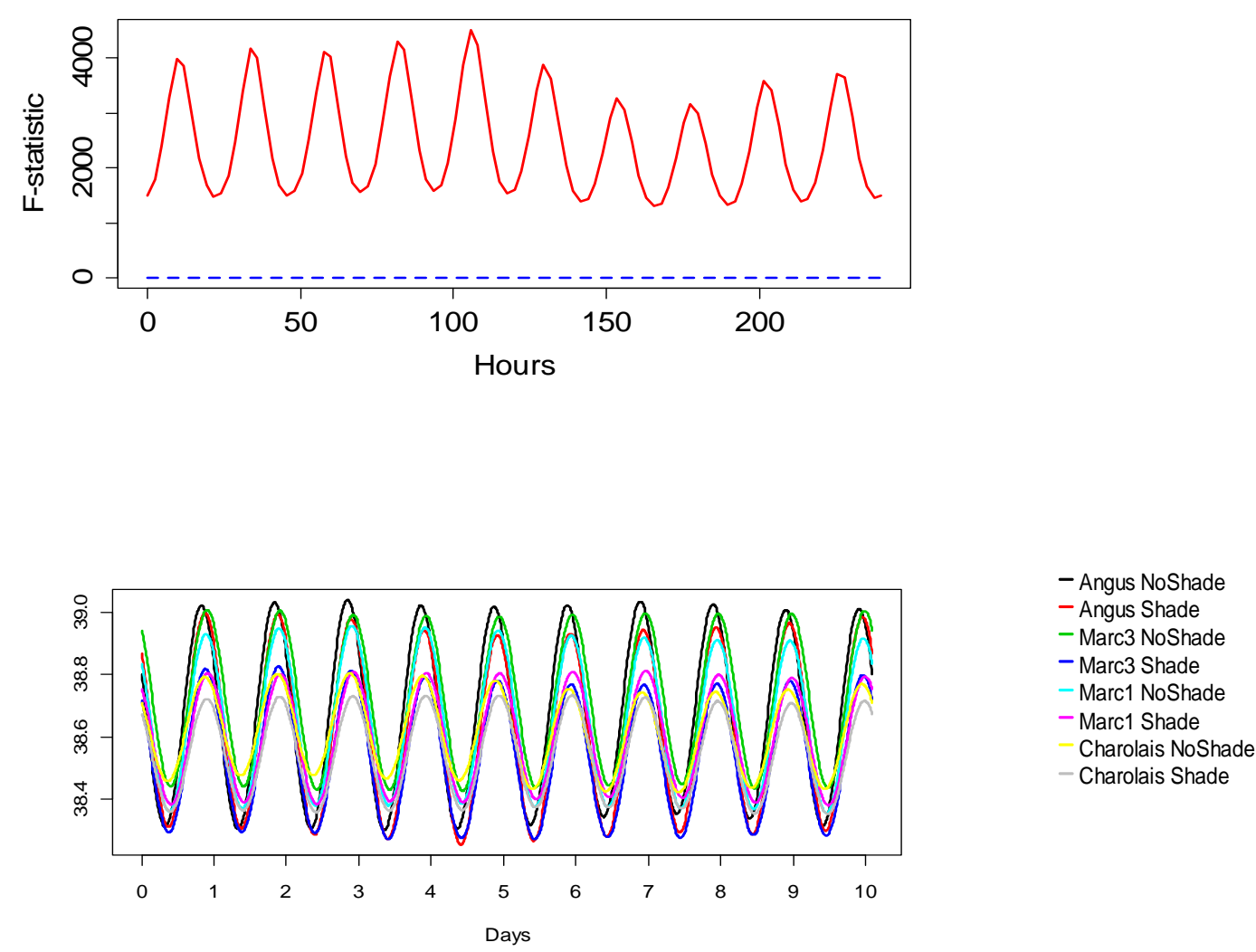

Since the interactions were significant, the simple effects of breads and shade were examined individually. Given shade, the $\mathrm{Tb}$ of all 4 breeds were significantly different over all days (Figure 6). Angus have the highest peak Tb and Charolais have the lowest peak Tb. In the middle, the peaks Tb of MARCIII and MARCI are close to each other. The amplitude of Tb produced by Angus was the largest and Charolais was smallest. However, given no shade, Tb of 4 breeds were not significantly different over all days (Figure 7) although the amplitude of Tb Charolais was still the smallest.

Given each breed, simple effects of shading were examined. For Charolais, no significant difference between shade and no shade was found (Figure 8). Surprisingly, the peak Tb of Charolais in no shade was a little bit higher than in shade pens. However, the overall performance of $\mathrm{Tb}$ of Charolais in shade was similar as in no shade. For MARCI, no significant difference between shade and no shade was found (Figure 9). Peak Tb of MARCI in no shade was higher than in shade pens, but the lowest Tb of MARCI in shade or no shade were close. For MARCIII, significant differences were found pointwise around day 2 and day 8 on (Figure 10). 
The overall predicted $\mathrm{Tb}$ of MARCIII in no shade was much higher than the predicted $\mathrm{Tb}$ in shade. For Angus, no significant difference between shade and no shade was found on (Figure 11). Although the predicted $\mathrm{Tb}$ of Angus in no shade was higher than the predicted $\mathrm{Tb}$ in shade, the difference was not as large as for MARCIII.

Figure 6. Functional $\mathrm{F}$ test for testing breeds differences given shade (top): observed $\mathrm{F}$ values -red, critical F values -- blue. Predicted Tb's of 4 breeds given shade (bottom): Angus - black; MARCIII - red; MARCI - blue; Charolais - white.
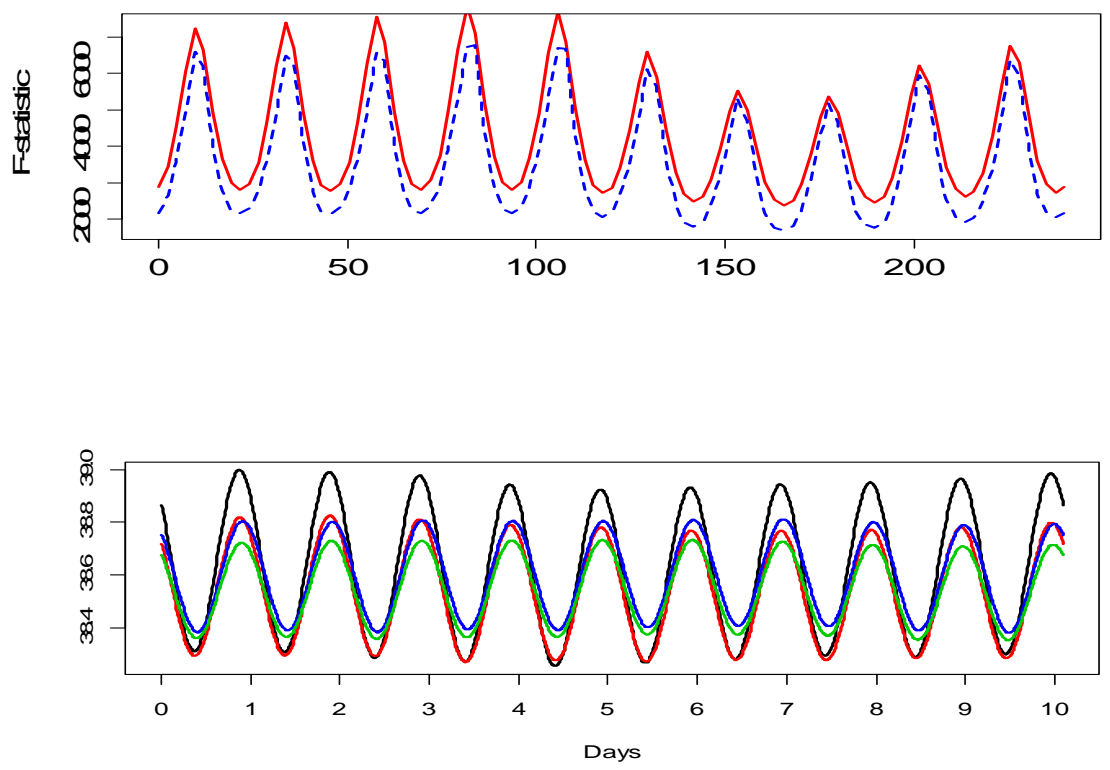
Figure 7.Functional $\mathrm{F}$ test for testing 4 breeds differences given no shade (top): observed $\mathrm{F}$ values -- red, critical F values -- blue.Predicted Tb's of 4 breeds given no shade (bottom):Angus black; MARCIII - red; MARCI - blue; Charolais - white.
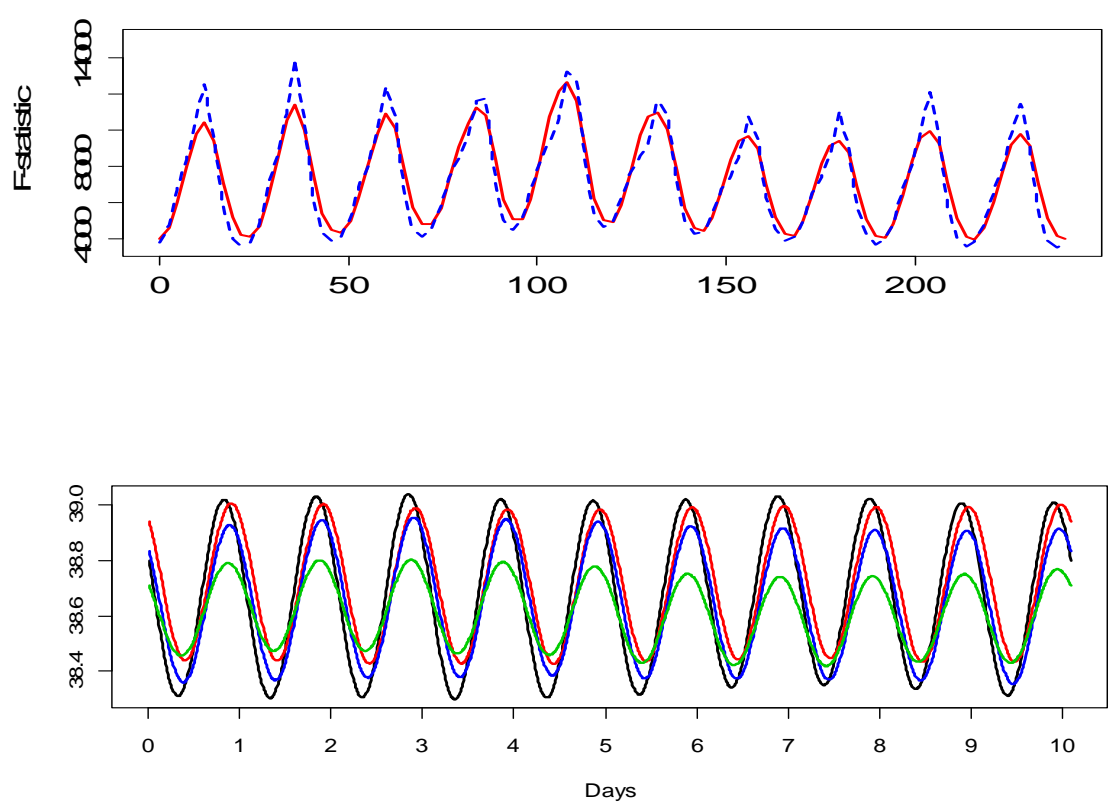
Figure 8. Functional t test for testing simple effect of shading given Charolais (top): observed $t$ values -- red, critical t values -- blue. Predicted Tb's of in shade and no shade given Charolais (bottom):Shade - black; No shade - red

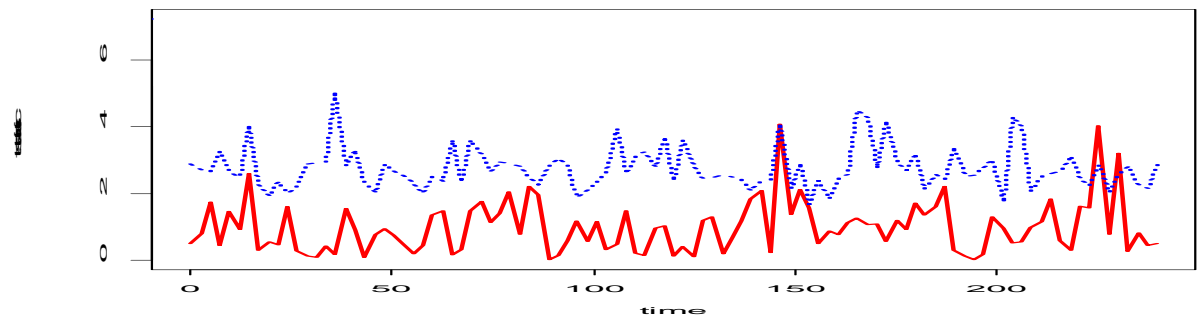

Prediction

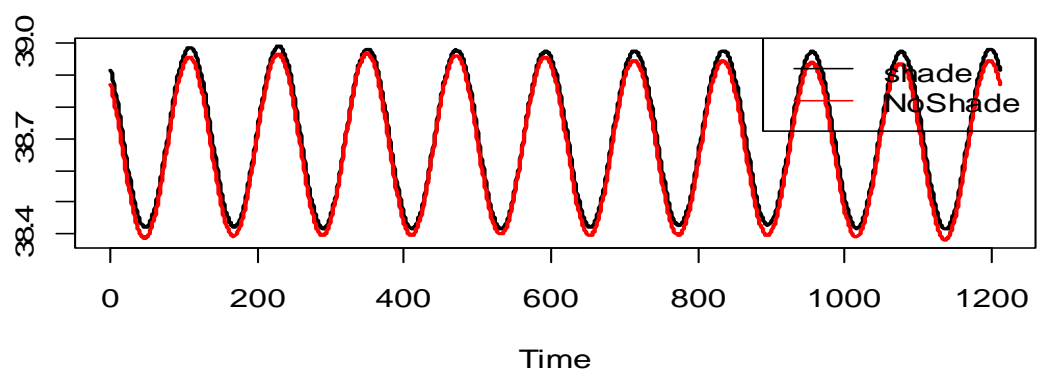


Figure 9. Functional $\mathrm{t}$ test for testing simple effect of shading given MARCI (top): observed $\mathrm{t}$ values -- red, critical t values -- blue. Predicted Tb's of in shade and no shade given MARCI (bottom):Shade - black; No shade - red

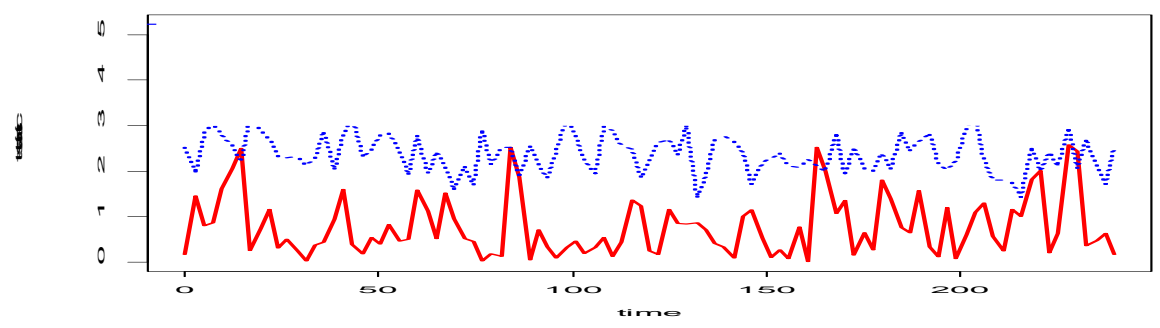

Prediction

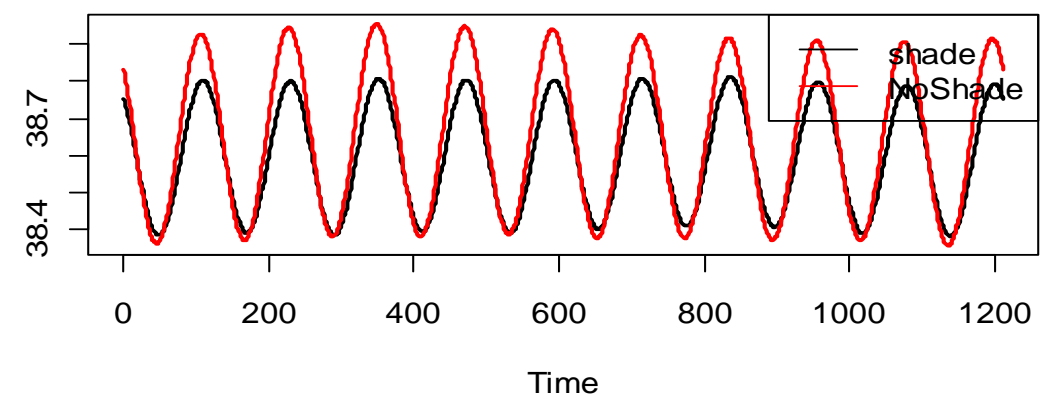


Figure 10. Functional $t$ test for testing simple effect of shading given MARCIII (top): observed $t$ values -- red, critical t values -- blue. Predicted Tb's of in shade and no shade given MARCIII (bottom):Shade - black; No shade - red

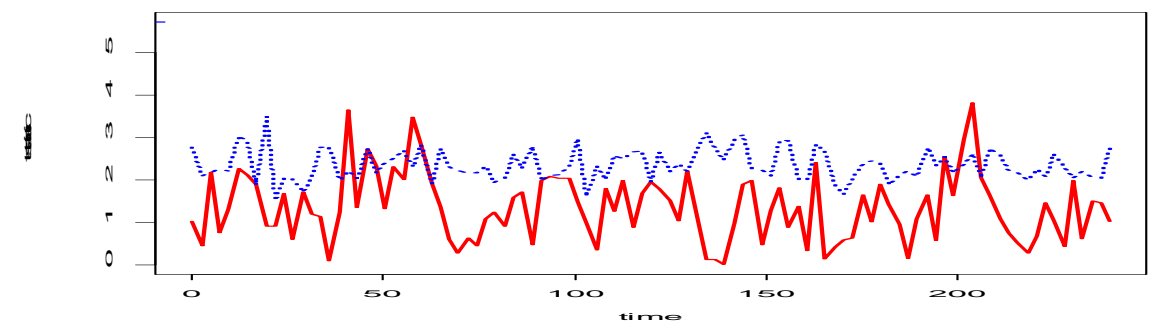

Prediction

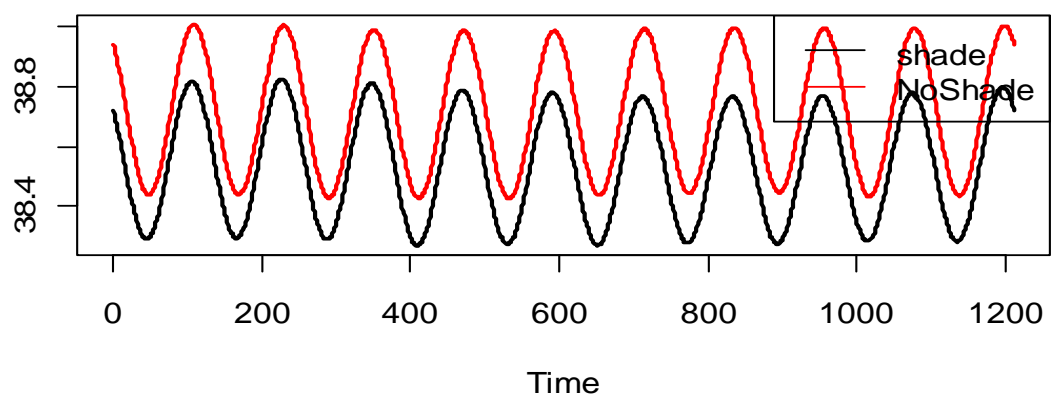


Figure 11. Functional t test for testing simple effect of shading given Angus (top): observed $t$ values -- red, critical t values -- blue. Predicted Tb's of in shade and no shade given Angus (bottom):Shade - black; No shade - red

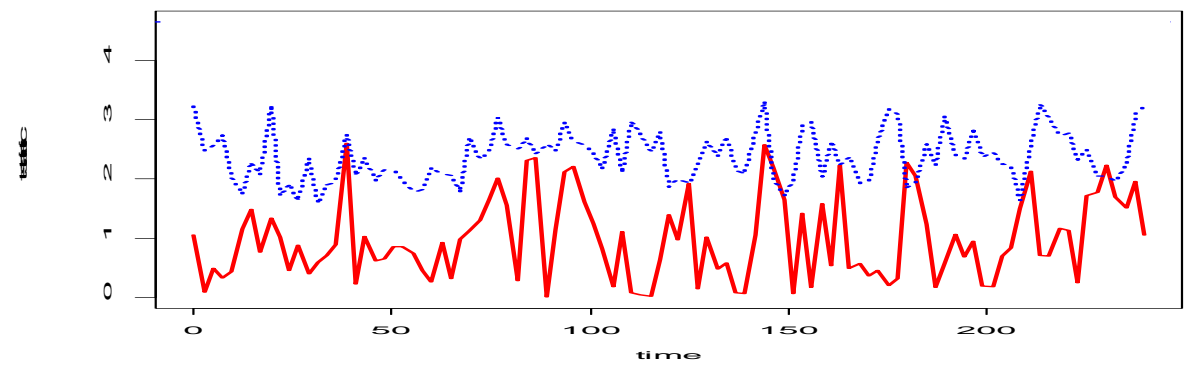

Prediction

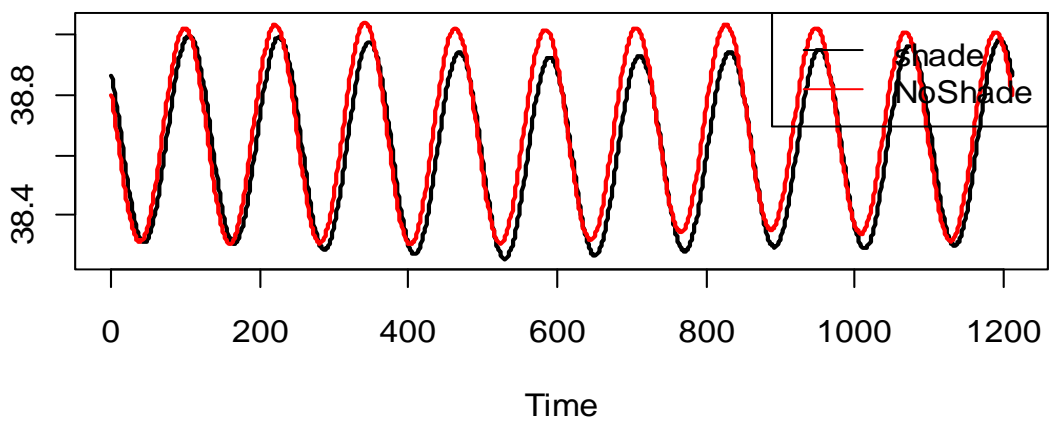

\subsection{Registration}

The registration was performed on all animals to align features for Tb curves. Figure 12 shows most of the animals have acceleration close to zero before hour 150 (i.e. day 6, Aug 1). It indicates Tb of most heifers were stable. Only two animals have large changes in acceleration around hour 75 (day 3-4), implying these two heifers experienced different trend of $\mathrm{Tb}$ from the others and they may have experienced extremely high $\mathrm{Tb}$ before the- heat stress days. After hour 150, acceleration of $\mathrm{Tb}$ by most of the heifers changed dramatically. It indicates heat stress has an effect on animals' Tb. The acceleration pattern changes showing $\mathrm{Tb}$ increased faster than usual. 
Figure 12.Registration: hourly acceleration of Tb over days for all 31 heifers.

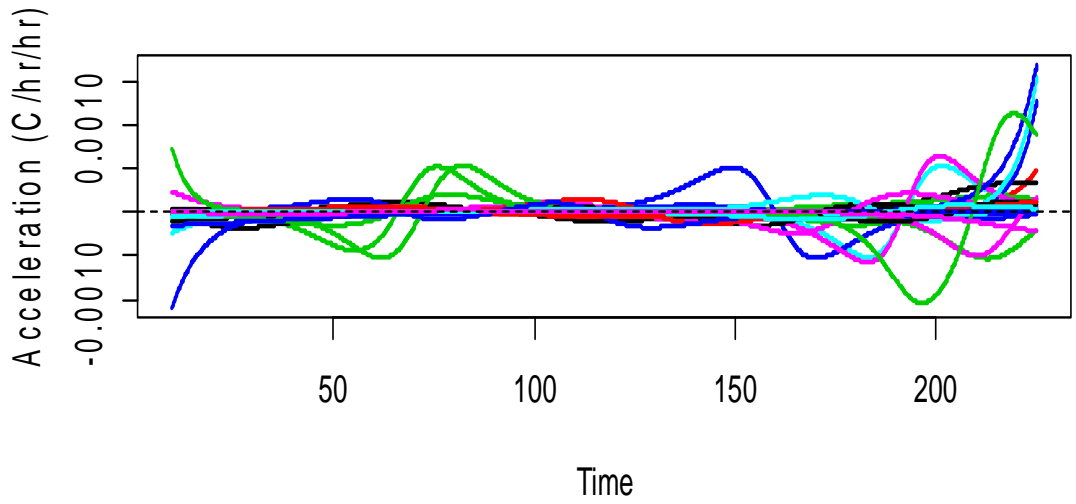

\subsection{Analysis on Heat Stress Days}

To mitigate the effect of the more thermoneutral days, the heat stress days were analyzed separately. Since heifers experienced a large change in $\mathrm{Tb}$ on heat stress, functional $\mathrm{F} / \mathrm{t}$ tests were constructed again on three heat stressed days. The result was the same as before. The shade*breed was significant (Figure 13). Significant simple effect can only be found on breeds given in shade over all 3 days. (Figure 14 left top) Angus have the largest amplitude on $\mathrm{Tb}$ while Charolais have the smallest. MARCIII and MARCI performed similar to each other (Figure 14 left bottom). Simple effect of breed was significant pointwise given no shade (Figure 14 right top). Heifers experienced significantly different peaks $\mathrm{Tb}$ during daytime; however, no difference in $\mathrm{Tb}$ was detectable at night (Figure 14 right top).

Figure 13. Functional $\mathrm{F}$ test for testing the breed *shade interaction on three heat stress days: observed t values -- red solid, critical t values -- blue dash

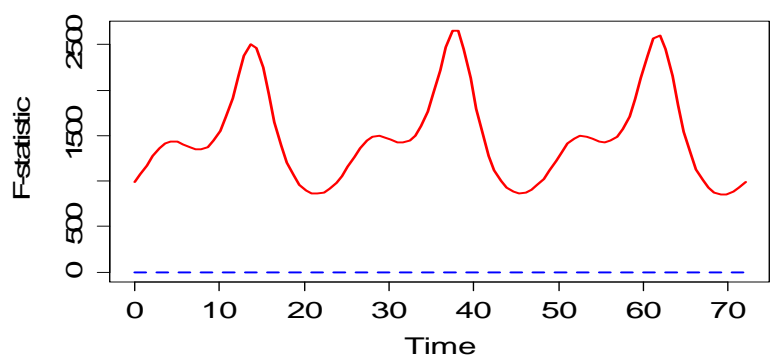


Figure 14. Functional $\mathrm{F}$ test for testing the simple effects of breeds given shade (left top) and given no shade (right top) on three heat stress days: observed F values -- red solid, critical F values -- blue dash; and the predicted Tb of breeds given shade (left bottom) and given no shade (right bottom) on three heat stress days: Angus - black; MARCIII - red; MARCI - blue; Charolais - white.
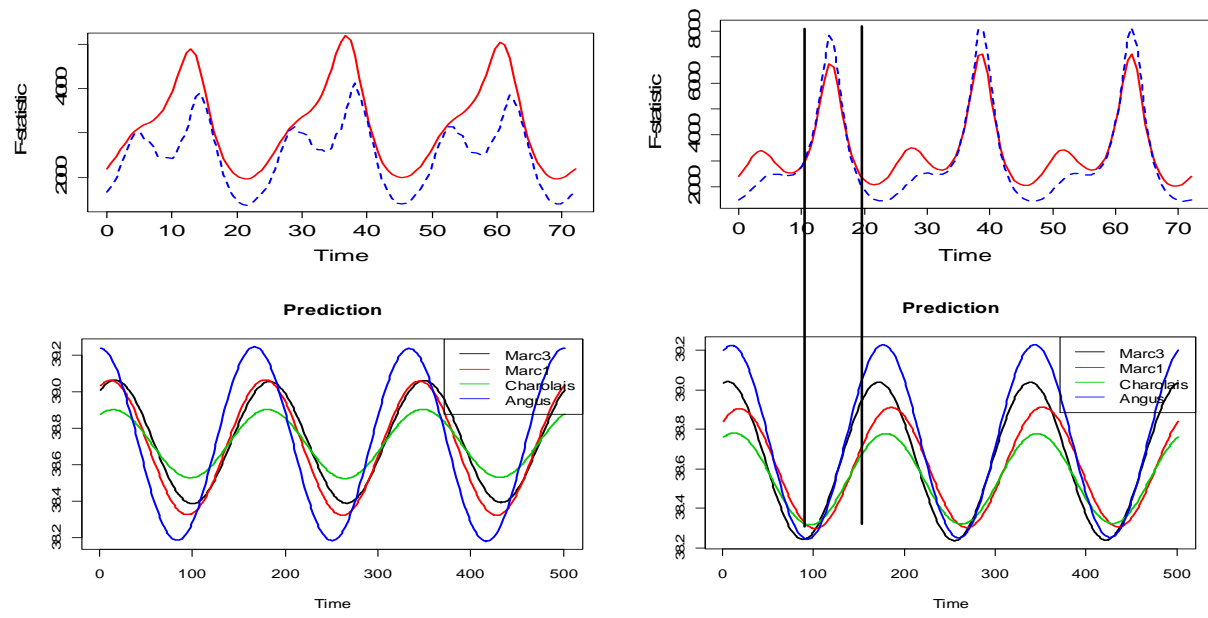

Figure 15. Functional $\mathrm{F}$ test for testing the simple effects of shade given each breed: observed $\mathrm{t}$ values -- red solid, critical t values -- blue dash
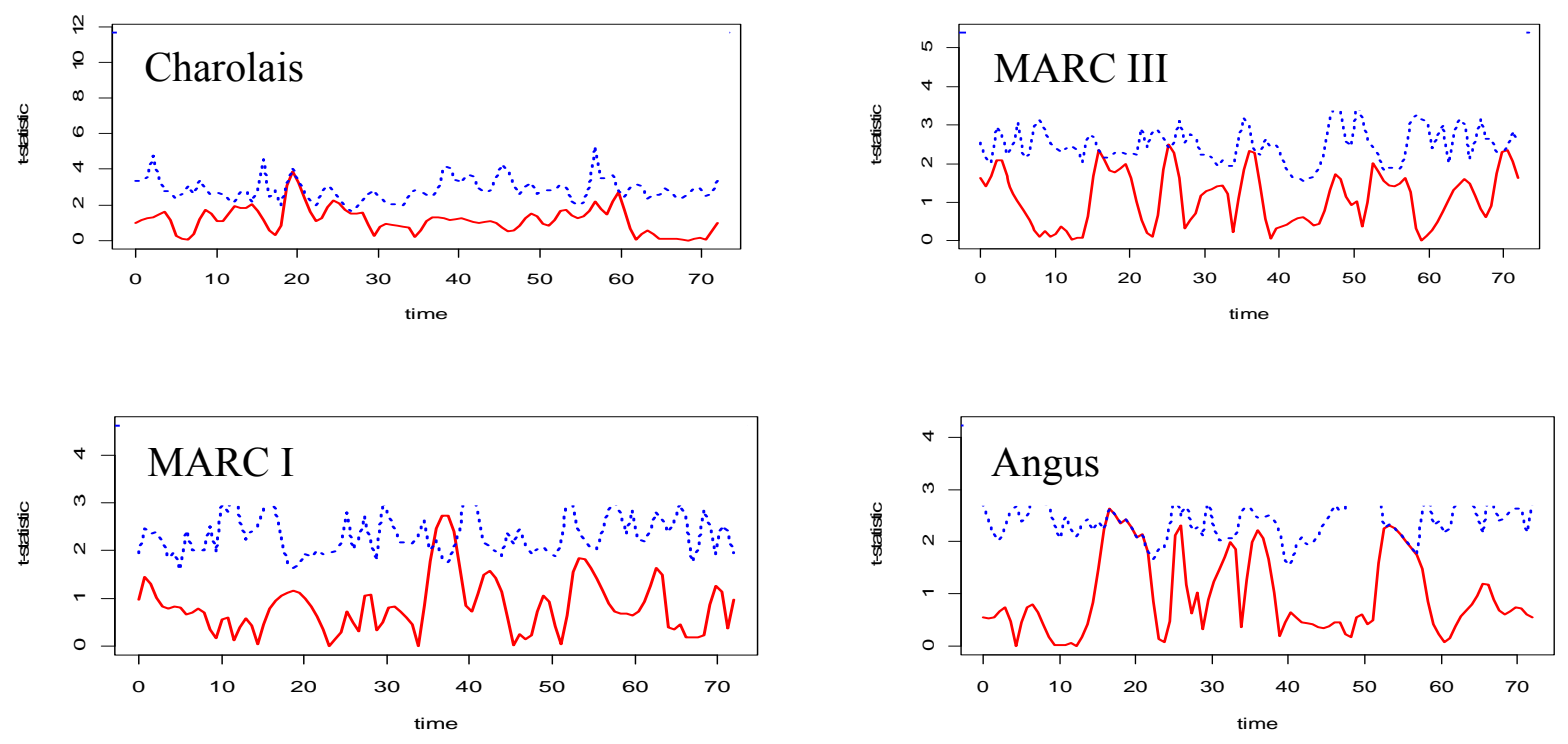

\section{CONCLUSION}


Functional data analysiscan be used to describe the dynamics of heifer $\mathrm{Tb}$ in an uncontrolled environment. After smoothing the data usinga roughness penalty, registration can be used to characterize the features of temperature curves and functional $\mathrm{F} / \mathrm{t}$ tests can be constructed to analyze treatment effects. Functional $\mathrm{F}$ test shows a significant interaction effects between breed and shade.Only one significant simple effect: differences in breed given shade was found.

Body temperature was sensitive to breed and shade. In each breed, heifers without shade will experience higher $\mathrm{Tb}$ than heifers with access to shade. Angus (black) has the most sensitive $\mathrm{Tb}$, while Charolais (white) has the least sensitive Tb. In daytime, Angus had the highest $\mathrm{Tb}$ and Charolais had the lowest $\mathrm{Tb}$ no matter whether there was a shade. However, the difference of $\mathrm{Tb}$ was not significant among breeds at night if there was no shade. Consequently, specific care is required for animals from different breeds during hot summer days. Breed and availability of shade interactions are important considerations when making management decisions regarding heat stress.

\section{References}

Craven, P., \& Wahba, G. (1978). Smoothing noisy data with spline functions. Numerische Mathematik, 31 (4), 377-403.

Gebremedhin, K., Hillman, P., Lee, C., Collier, R., Willard, S., Arthington, J., et al. (2008). Sweating rates of dairy cows and beef heifers in hot conditions. Transactions ASABE, 51, 21672178.

O, J. J., Hooker, G., \& Graves, S. (2009). Functional data analysis with R and MATLAB. Springer. 